\title{
26. Geschichte von Dialogformen
}

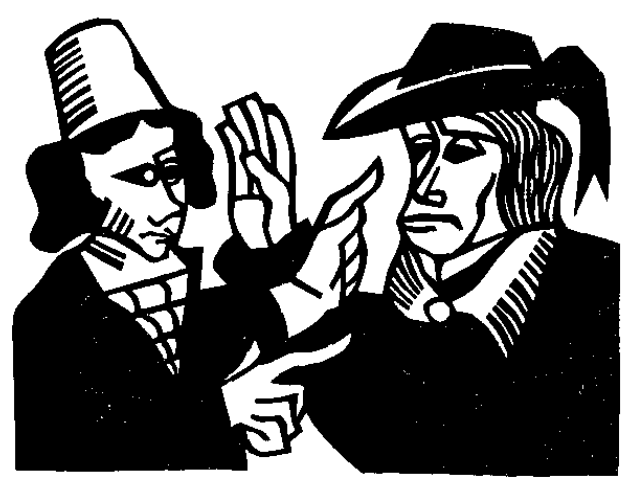

1. Fragestellungen und Forschungslage

2. Zur Frage der Quellen

3. Aspekte von Dialogformen und ihre Geschichte

3.1 Sprachliche Handlungsmuster, Sequenzmuster und Strategien

3.2 Institutionen und Sprecherkonstellationen

3.3 Äußerungsformen

3.4 Themen und thematische Zusammenhänge

3.5 Kommunikationsprinzipien

4. Literaturhinweise

\section{Fragestellungen und Forschungslage}

Um einen ersten Eindruck von den Aspekten einer historischen Betrachtung von Dialogformen zu geben, sollen hier einige Fragen formuliert werden, deren Beantwortung zu den Aufgaben einer Geschichte von Dialogformen gehört:

(i) Welche Dialogformen lassen sich in bestimmten Gruppen, Gesellschaften oder Kulturen zu bestimmten Zeiten beobachten? Man könnte hier vom „kommunikativen Haushalt" einer Gesellschaft sprechen (vgl. Luckmann 1986, 206). Inwiefern werden diese Dialogformen von den Sprechern als solche wahrgenommen (,members' categories“ im Gegensatz zu Artefakten der wissenschaftlichen Beschreibung)?

(ii) Lassen sich Schwankungen im Anteil dialogischer und monologischer Umgangsformen in bestimmten Praxisbereichen beobachten? (Zum Bereich der Logik/ Dialektik vgl. Ong 1958; Hamblin 1970; ein weiteres Beispiel: traditionelle Verkaufsgespräche vs. nicht- oder reduziert-dialogische Organisation des Kaufens im Supermarkt.)

(iii) Wie kann man eine uns nicht unmittelbar vertraute Dialogform besser verstehen? In diesem hermeneutischen Aspekt besteht ein enger Zusammenhang mit Fragestellungen der Ethnographie (vgl. etwa Geertz 1983), insbesondere der Ethnographie der Kommunikation (vgl. Beiträge in Gumperz/Hymes 1972 oder Bauman/ Sherzer 1974).

(iv) Wie lassen sich befremdliche Aspekte unserer eigenen Dialogformen erklären? 
(Z. B. durch den Blick auf ältere historische Traditionen, die zu uns heranreichen. Beispiel: Höflichkeitsrituale.)

(v) Wie entstehen neue Varianten von Dialogformen? (Z.B. durch Bevorzugung bestimmter Verlaufstypen einer etablierten Dialogform.)

(vi) Wie spielen sich neue Varianten einer Dialogform in einer bestimmten Gruppe ein (Wiederholung eines Präzedenzverlaufs, Standardisierung, Konventionalisierung)? (Das Sich-Einspielen von Sonderformen von Dialogverläufen kann man bisweilen in Kleingruppen wie Familien oder Freundeskreisen unmittelbar beobachten.)

(vii) Wie und warum verbreiten sich Neuerungen im Bereich der Dialogformen (spontane Nachahmung, Lehren und Lernen, Missionierung; funktionale Vorteile einer Neuerung, Prestige der „Erfinder“ der Neuerung; die Rolle von sozialen „networks")?

(viii) Wie entwickeln sich Dialogformen unter spezifischen institutionellen und medialen Bedingungen (die Huldigung, das Interview)?

(ix) Gibt es Aspekte von Dialogformen, die leicht Veränderungen unterliegen bzw. solche, die eher veränderungsresistent sind? (Elementare illokutionäre Sequenzmuster wie Frage/Antwort, Behaupten/Bestreiten, Grundmuster von Aufforderung/Weigerung - im Gegensatz etwa zu den spezifisch höflichen Varianten scheinen relativ konstant zu bleiben. Dagegen unterliegen Äußerungsformen, Themen und Kommunikationsprinzipien stärker Veränderungen, desgleichen institutionelle Sonderformen von Dialogen.)

(x) Gibt es Dialoguniversalien (die Befolgung von Rationalitätsprinzipien, Höflichkeitsprinzipien; das Vorhandensein von elementaren Mustern für sprachliche Handlungen wie das Behaupten oder Teilhandlungen wie das Referieren)?

(xi) Lassen sich in einzelnen Gruppen oder Gesellschaften in bestimmten Zeiträumen größere Tendenzen in der Entwicklung von Dialogformen erkennen, und wie kann man diese beschreiben (Standardisierung, Ritualisierung, Spezialisierung, Ausdifferenzierung, Säkularisierung, Zivilisierung)?

(xii) Lassen sich für bestimmte Bereiche des dialogischen Redens epochenartige Abschnitte beschreiben, die durch spezifische gesellschaftliche Bedingungen gekennzeichnet sind?

(xiii) Aus welchen Bausteinen bestehen Erklärungen der Kontinuität und der Veränderung von Dialogformen? (Z.B. Hinweise auf: Ziele und Interessen einzelner Sprecher; die Bedingungen, unter denen diese handeln; die Prinzipien, denen sie folgen; das schon vorhandene Repertoire an Dialogformen; die Zwecke, denen die alten und neuen Dialogformen dienen; die kollektiven Konsequenzen individueller Handlungen.)

(xiv) In welcher Weise lassen sich historische Untersuchungen als Prüfsteine für Dialogtheorien nutzen? (Z.B. in bezug auf den Status des Regelbegriffs, die Beschreibung von Graden der Konventionalisierung oder die Frage der Universalität von Kommunikationsprinzipien; vgl. Art. 10.)

Die Forschungslage zur Geschichte von Dialogformen muß differenziert beurteilt werden. Einerseits gibt es vielfältig verstreute Arbeiten zu einzelnen historischen Dialogen und Dialogformen, vor allem aus den Bereichen der Literaturgeschichte, der Rhetorikgeschichte und der Philosophiegeschichte. Diese sind aber kaum systematisch erfaßt, so daß es schwierig ist, einen allgemeinen Überblick über den Stand der Forschung in diesem Sektor zu gewinnen. In Arbeiten dieser Art stehen im allgemei- 
nen folgende Forschungsinteressen im Vordergrund: hermeneutische Fragen, Fragen der literarischen bzw. rhetorischen Technik der Dialogdarstellung, Fragen der Struktur einzelner literarischer Werke bzw. der Besonderheiten einzelner Autoren. Entwicklungsgeschichtliche Untersuchungen im eigentlichen Sinne sind eher selten. Brauchbare Ansätze finden sich, um nur einige Beispiele zu nennen, in Hirzel (1895) oder in neueren Arbeiten wie der kurzen Einleitungsskizze in Schmölders (1986) und den der Rhetorik verpflichteten Darstellungen in Göttert (1988) und Beetz (1990). Ein besonderes Desideratum sind Arbeiten, die eine explizite dialogtheoretische Fundierung mit einer entwicklungsgeschichtlichen Perspektive verbinden und eine Übersicht über die historische Entwicklung grundlegender Dialogformen geben. Seit einigen Jahren zeigt sich zwar verstärkt ein historisches Interesse in der Dialogforschung (vgl. z. B. Henne 1980; v. Polenz 1981; Schlieben-Lange 1979; 1983; Weigand 1988), aber in diesen Arbeiten, die sich an neueren Entwicklungen im Bereich der Sprechakttheorie und Gesprächsanalyse orientieren, steht noch die Analyse von einzelnen sprachlichen Handlungen oder Dialogen in Texten älterer Sprachstufen im Vordergrund. Die Perspektive dieser Arbeiten ist zumeist kontrastiv, zumindest implizit, d.h. es werden die in bestimmten historischen Dialogdarstellungen erkennbaren Dialogmuster verglichen mit der heutigen Dialogpraxis. Die eigentliche Entwicklung der Dialogformen sowie deren Zusammenhänge und Bedingungen werden (noch) nicht behandelt. Was eine Geschichte von Dialogformen mit einer genuin evolutionären Perspektive und systematischen Fragestellungen angeht, so befinden wir uns noch ganz am Anfang der Forschung. Dementsprechend dokumentiert der vorliegende Artikel einerseits vorhandene Ansätze, andererseits bietet er in vielen Punkten erst noch einzulösende Programmatik und die Formulierung von Desideraten.

An dieser Stelle sollen einige Grundannahmen einer Theorie der Entwicklung von Dialogformen dargestellt werden. Eine derartige Theorie soll systematische Fragestellungen der zu Beginn dieses Abschnitts erwähnten Art eröffnen, wie wir sie auch aus anderen sprachhistorischen Arbeitsfeldern kennen. Diese Annahmen betreffen zum einen die Dialogtheorie, zum andern die entwicklungsgeschichtliche Perspektive.

Die wesentlichen dialogtheoretischen Annahmen lassen sich folgendermaßen zusammenfassen: Historische Dialogformen sind charakterisiert durch bestimmte Ausprägungen ihrer grundlegenden Organisationsprinzipien (vgl. Art. 10), z.B. der in entsprechenden Dialogen realisierten Sequenzmuster, der charakteristischen Äußerungsformen, der thematischen Zusammenhänge und der in den betreffenden Dialogen angewendeten Kommunikationsprinzipien. In jedem der Aspekte der Grundstruktur einer Dialogform können unter geeigneten Bedingungen Veränderungen eintreten. Veränderungen in einem Aspekt sind häufig mit Veränderungen in anderen Aspekten verknüpft oder bringen solche hervor. So hängen Veränderungen im Wortschatz häufig mit Veränderungen im thematischen Bereich zusammen, oder Veränderungen in der Gültigkeit von Kommunikationsprinzipien führen zu einer neuen Bewertung von bestimmten Dialogstrategien.

Die entwicklungsgeschichtliche Perspektive läßt sich folgendermaßen darlegen: Eine über eine gewisse Zeit hin stabile Dialogform kann man, in Anlehnung an die 
Analyse des Konventionsbegriffs in Lewis (1969), als die Lösung einer rekurrenten kommunikativen Aufgabe deuten. So könnte man z.B. das Feilschen um den Preis einer Ware als eine mögliche Lösung des Problems auffassen, eine Einigung über einen zunächst nicht klar fixierten Preis zu erzielen, oder die uns vertraute alltägliche Form von Frage/Antwort-Dialogen könnte man verstehen als eine klassische Lösung des Problems der Gewinnung gemeinsamen Wissens. Wenn nun eine neue Problemsituation eintritt, ergibt sich für die Mitglieder einer Gesellschaft oder einer sozialen Gruppe die Aufgabe, adäquate Lösungen zu finden. Am Beispiel der Gewinnung (zumindest partiell) gemeinsamen Wissens könnte man zeigen, wie für spezielle Problemsituationen Speziallösungen entdeckt, aufgenommen und verbreitet wurden, z. B. die Dialogformen des Verhörs, der Beichte oder des Interviews. Es entstehen in diesen Fällen institutionsspezifische Sonderformen des ursprünglichen Frage/Antwort-Musters, die bestimmte Zwecke in diesen Institutionen erfüllen (vgl. Art. 12). Weitere Beispiele für diese Betrachtungsweise sind: In welcher Weise haben Sprecher gelernt, Schwierigkeiten wie Mißbehagen und Mißtrauen bei der Begegnung mit Fremden zu überwinden (z. B. durch höfliche Rituale wie Grüße und Komplimente; vgl. Beetz 1990, 168ff.) oder Konflikte zu entschärfen, die durch eine Verfehlung eines der Dialogpartner entstanden sind? (Etwa durch Wiedergutmachungshandlungen wie Entschuldigungen, deren Funktion in „remedial interchanges“ Goffman 1971, 124ff. beschrieben hat.) Eine Aufgabe einer theoretisch fundierten Geschichte von Dialogformen könnte es nun sein zu zeigen, wie die betreffenden kommunikativen Verfahrensweisen, samt Äußerungsformen, Strategien und Dialogprinzipien, in Anpassung an die ökologischen Forderungen wechselnder Problemsituationen entstehen und schrittweise verändert werden (vgl. Toulmin 1972, 352). Die hier skizzierte Problemlöseauffassung der Genese, Veränderung und Kontinuität von Dialogformen ist ein nützlicher Analyserahmen, der dazu beiträgt, die Geschichte der Dialogformen im Zusammenhang zu sehen mit den kommunikativen Zwecken, die Dialogformen in einem bestimmten Umfeld erfüllen. Nun ist aber die eben gegebene Beschreibung dieser Auffassung zumindest in zweierlei Hinsicht einschränkungsbedürftig. Erstens darf die Idee des Problemlösens nicht zu aktivistisch verstanden werden. Veränderungen in Dialogformen werden zumeist nicht von einzelnen Individuen oder Gruppen intentional oder gar reflektiert eingeführt. Vielmehr scheint es so zu sein, daß sich bei den tastenden Versuchen der Dialogteilnehmer, mit lokalen praktischen Problemen fertig zu werden, neue kommunikative Varianten ergeben, von denen manche nach bestimmten Bewertungsprinzipien besonders attraktiv sind und deshalb wieder aufgenommen werden, sich einspielen und verbreiten, ohne daß die ursprünglichen „Entdecker“ die Intention gehabt hätten, eine neue Dialogform zu etablieren (vgl. Ullman-Margalit 1978; Keller 1990). Zweitens darf man nicht erwarten, daß für alle Dialogformen einer hochentwikkelten kommunikativen Praxis eine direkte funktionale Erklärung des erwähnten Typs möglich ist. Das könnte z. B. gelten für die Entdeckung spielerischer Varianten etablierter Dialogformen oder für überkommene ,leere Rituale“. Bei spielerischen Formen wie der parodistischen Dialognachahmung, dem Rätsellösedialog oder dem Erfinden von Geschichten könnte man sich eine Genese denken, die nicht aus der kommuni- 
kativen Not, sondern aus der Freude am kommunikativen Luxus zu erklären ist. Hier könnte es schwer werden, vorgegebene Probleme anzugeben, zu deren Lösung diese Dialogformen hätten beitragen sollen, was aber nicht bedeutet, daß diese Formen nach ihrer Entdeckung nicht charakteristische kommunikative Zwecke erfüllen. Etwas anders gelagert ist der Fall, in dem eine ursprünglich problemlösende Dialogform als „leeres Ritual“" weitertradiert wird. In diesem Fall bewirkt ein besonders konservativer Selektionsprozeß die Ablösung der Form von ihrem primären Praxiszusammenhang. Auch hier ist eine direkte funktionale Erklärung nicht möglich (vgl. Toulmin 1972, 350f.).

$\mathrm{Ob}$ die für eine evolutionäre Betrachtung notwendigen Fakten jeweils zugänglich sind, ist natürlich eine Frage der Quellenlage. Für die elementaren Dialogformen, deren Entstehung vor die Zeit verfügbarer Quellen fällt, kann es hilfreich sein, in Form einer Konjekturalgeschichte die Struktur der Zusammenhänge zu rekonstruieren, in denen diese Dialogformen entstanden sein könnten. Schöne Beispiele für die Form einer derartigen Konjekturalgeschichte finden wir in Strecker (1987).

\section{Zur Frage der Quellen}

Die ideale Quellenlage für die Beschreibung einer Dialogform könnte man folgendermaßen charakterisieren: Man besitzt zahlreiche Videobänder (samt Transkriptionen) von entsprechenden Dialogen, deren Aufnahme man teilnehmend beobachtet hat, und man ist durch eigene Praxis mit der betreffenden Dialogform vertraut. Gemessen an diesen Idealbedingungen muß man bei der Beschreibung von historischen Dialogformen zumeist weitgehende Abstriche machen, die für die Vertrauenswürdigkeit der Beschreibungen natürlich Konsequenzen haben. Dem genannten Ideal käme man etwa dann noch relativ nahe, wenn man als Zeitgenosse die Geschichte von Dialogformen in den Medien auf der Grundlage von authentischem Bandmaterial schreiben wollte. Eine ungewöhnliche Variante dieses Quellentyps sind Beethovens Konversationshefte, in die die Besucher des schwerhörigen Komponisten ihre Gesprächsbeiträge eintrugen. Einen Schritt weiter entfernt vom authentischen Material sind Quellen wie Protokolle, z. B. von Parlamentssitzungen (vgl. Grünert 1974; Holly 1982), Reichstagen, Gerichtsverfahren (fürs Mittelalter z. B. sog. Urgichtenbücher, d.h. Geständnisbücher), Dialogen mit Kindern (wie sie in der frühen Kindersprachforschung vorliegen, z. B. Katz/Katz 1928) etc. Es folgen als Quellen die Beschreibungen von Dialogverläufen, die wir in Briefen, Tagebüchern etc. finden. Weiterhin Darstellungen von exemplarischen Dialogverläufen in historischen, philosophischen, literarischen, pädagogischen und dialogkritischen Werken. Einige klassische Beispiele für diesen Quellentyp: die Beratungen der Griechen im „Peloponnesischen Krieg“ des Thukydides, der „conseil des barons“ im altfranzösischen „Rolandslied“ (vgl. Köhler 1968), die Beratungsgespräche im Thronrat in Opitzens „Argenis“-Übersetzung; Lehrgespräche (zwischen Trevrizent und Parzival) bei Wolfram von Eschenbach (vgl. Kästner 1978), 
in den „Colloquia familiaria“ des Erasmus und anderen sog. Schülergesprächen (vgl. Bömer 1897/1899), (zwischen Mentor und Telemach) in Fénelons „Télémaque“ oder in Campes „Robinson der Jüngere“ (vgl. Naumann 1991); Vorwurfssequenzen im Ehebüchlein des Albrecht von Eyb; Streitszenen in Fastnachtsspielen, Streit- und Gerichtsdialoge im „Nibelungenlied“ oder im „Ackermann von Böhmen“. Weiterhin gibt es Darstellungen von Dialogverlaufstypen in Lehrbüchern (z. B. in sog. Gesprächsbüchern und anderen Fremdsprachenlehrbüchern), Kodifizierungen von hervorgehobenen Verlaufstypen in Rechts- und Verwaltungstexten, Normierungshinweise für Dialogverläufe in Verhaltenslehren (z. B. Knigge 1790), Komplimentierbüchern, Konversationslehren (z. B. Hunold 1716), mittelalterlichen Tischzuchten etc. (zu sprachreflexiven Quellen vgl. Gloning 1993). Die meisten dieser Quellen sind zwei bis drei Stufen von authentischen Dialogen entfernt, so daß wir bei der Einschätzung der Quelle Gesichtspunkte wie das Wissen und die Darstellungsintentionen des Autors, die Überlieferungslage der betreffenden Quelle und viele andere, vor allem hermeneutische Fragen berücksichtigen müssen. Wie diese Beispiele für Quellentypen zeigen, besteht ein enger Zusammenhang zwischen der Geschichte der Dialogdarstellungen bzw. Dialogtyp-Darstellungen und der Geschichte von Dialogformen (vgl. Schlieben-Lange 1979; Betten 1985; s. auch Art. 25).

\section{Aspekte von Dialogformen und ihre Geschichte}

\subsection{Handlungsmuster, Sequenzmuster und Strategien}

Im vorliegenden Abschnitt 3 soll der für eine Geschichte von Dialogformen leitende dialogtheoretische Gedanke näher ausgeführt werden, daß sich Dialogformen in allen Aspekten ihrer Grundstruktur verändern können und daß Veränderungen in unterschiedlichen Aspekten miteinander verknüpft sein können.

Grundlegende Fakten in der Geschichte von Dialogformen sind die Einführung und die Veränderung, aber auch die Konservierung von einzelnen Handlungsmustern, ganzen Sequenzmustern und besonderen Strategien innerhalb der Sequenzmuster. Streng genommen bedeutet jede Einführung eines neuen Handlungsmusters bzw. die Veränderung eines solchen die Veränderung des ganzen Sequenzmusters und damit der Dialogform. In spieltheoretischer Redeweise könnte man sagen, daß sich das ganze Spiel verändert, wenn eine neue Zugmöglichkeit eingeführt wird (vgl. Art. 8). Wenn man die Betrachtung einzelner prominenter Handlungsmuster zum Ausgangspunkt nimmt, was durchaus sinnvoll sein kann, so muß man darauf achten, daß man die Zusammenhänge dieser Handlungsmuster adäquat erfaßt. Als Beispiel könnte man die Veränderungen des Musters BEWEISEN im Laufe der mittelalterlichen und frühneuzeitlichen Prozeßpraxis untersuchen (vgl. Heringer 1977, Kap. 4; Fritz/Muckenhaupt 1984, Kap. 26). BEWEISEN kann man auffassen als einen Reaktionszug im Vorwurfsoder Anklagespiel, der durch besondere Ausführungsmöglichkeiten und -beschränkungen definiert ist. So kann man z. B. nach den Rechtsvorschriften des Sachsenspie- 
gels beim Vorwurf des Raubs seine Unschuld dartun, indem man einen Reinigungseid schwört. Dies ist aber nur zulässig, wenn man nicht des wiederholten Raubs angeklagt wird. Sollte letzteres der Fall sein, kann man seine Unschuld dartun, indem man „das glühende Eisen trägt", „,in einen wallenden Kessel greift" oder sich dem Zweikampf stellt. Entscheidend ist nun, daß der Historiker die verschiedenen Beweisformen, darunter auch die sog. Gottesurteile, die ja wesentlich nichtsprachliche Handlungen sind, im Zusammenhang ihres Handlungsumfeldes (Bedingungen, Alternativen, Konsequenzen) beschreibt. Aufgabe der entwicklungsgeschichtlichen Betrachtung ist es, Veränderungen in den Beweismitteln (z.B. die Abschaffung der Unschuldseide und die Aufwertung des Geständnisses im Strafprozeß der Neuzeit) und in den Zusammenhängen des Beweises zu rekonstruieren (z. B. die Verteilung der Beweislast oder die Rolle der Folter; vgl. Kornblum 1971). Wenn man in diesem Beispiel von der Geschichte des Beweisens spricht, so muß man allerdings beachten, daß handlungskennzeichnende Ausdrücke wie mhd. bewisen einem Bedeutungswandel unterliegen können, so daß man sich nicht darauf verlassen kann - wie oben zunächst naiv getan -, daß ein Sprecher des Mhd. sich mit der Verwendung von bewisen oder bewisunge auf ein Muster BEWEISEN im modernen Sinne bezieht. Die sprechaktkennzeichnenden Ausdrücke sind hier nur ein erster Hinweis für eine historische Fragestellung. ( $\mathrm{Zu}$ sprechaktkennzeichnenden Ausdrücken in mhd. literarischen Texten vgl. v. Polenz 1981; zum Problem des Verstehens fremder Lebensformen vgl. Winch 1972; vgl. auch Art. 9.)

Am Beispiel der Formen der LIEBESERKLÄRUNG, für die Schwarz (1984) den Versuch einer „Sprechaktgeschichte“ vorgelegt hat, läßt sich nochmals verdeutlichen, $\mathrm{da} ß$ es nicht primär das isolierte Sprechaktmuster ist, für das aufschlußreiche historische Einsichten zu erwarten sind, sondern die weiteren Sequenzzusammenhänge. Führt man sich vor Augen, in welchen Aspekten Veränderungen der Praxis von Liebeserklärungen zu erwarten sind, dann kommt man u. a. zu folgenden Fragestellungen: Wer darf das Spiel der Liebeserklärung eröffnen und mit welchen Mitteln? Welche Teilschritte gibt es für eine Liebeserklärung? Welche Reaktionsmöglichkeiten gibt es auf die einzelnen Teilzüge einer Liebeserklärung? Wie kann man eine abschlägige Reaktion angemessen gestalten? Welche strategischen Probleme und Alternativen gibt es für die beiden Beteiligten (Probleme der Offenlegung der Gefühle und der Festlegung auf bestimmte Handlungskonsequenzen)? Welche Folgen haben die betreffenden Handlungen jeweils? In diesen Aspekten wird man fundamentale Unterschiede zwischen einer Liebeserklärung bei mittelalterlichen Adligen, viktorianischen Bürgern und heutigen Studenten/Studentinnen erwarten, Unterschiede, die viel über die jeweilige Lebensform erkennen lassen. Diese Aspekte erfaßt man nicht, wenn man einzelne Sprechaktmuster isoliert betrachtet.

Als Beispiele für Sequenzmuster, deren Form und Status historische Veränderungen erfahren haben, seien nur zwei genannt, Streitdialoge und Komplimentierdialoge. Es gibt z. B. einen Typ von Herausforderungsdialog, den man in ähnlicher Form sowohl bei den Homerischen Helden als auch bei den germanischen Kriegern findet. Als Vorbereitung zum Kampf loben sich die Kämpfer selbst und beschimpfen sich 
wechselseitig (vgl. Bebermeyer 1984; Bax 1991). Für diese Art der Reizrede bzw. für den aus solchen Reizreden bestehenden Dialog gibt es in den germanischen Dialekten sogar besondere redekennzeichnende Ausdrücke: im Altnordischen hvöt (,Aufreizung") und senna (,Wortstreit“) im Altenglischen gylpcwide oder gylpspraec (,Prahlrede") (Beowulf 981), im Mittelhochdeutschen das mit gylp verwandte gelpf (Nibelungenlied 430,1). Dieses Muster in seiner ritualisierten Form hat $\mathrm{m}$. W. in den heutigen mitteleuropäischen Kulturen nicht den Status, den es in Kulturen hatte, in denen der Einzelkampf eine wichtige Rolle als Entscheidungsverfahren spielen konnte. Es finden sich jedoch entfernt verwandte Muster im Streit unter Kindern oder auch in Wahlkampfdiskussionen von Politikern. Diese Dialogformen gehören in den größeren Rahmen der Streitkommunikationen, für deren Geschichte wir zwar vielfältige Einzelbeobachtungen besitzen, aber keine Übersichtsdarstellung. Hier müßten typische, zeitspezifische Streitgegenstände beschrieben werden (vom Besitzstreit der germanischen Gesetze über Rangstreitigkeiten bei den Reichstagen der frühen Neuzeit bis hin zu modernen Beziehungskonflikten), weiterhin die unterschiedlichen Realisierungen von Mustern wie Vorwerfen, Beschimpfen, Verspotten, Hänseln, „Raillerie“ (vgl. Hunold 1716, Kap. VI; s. a. Berger 1978, 226f.), Beleidigen, Polemisieren (vgl. Schwitalla 1986), die typischen Reaktionsmuster (z. B. Beleidigung/Gegenbeleidigung, bes. in Formen ritueller Beleidigung; vgl. Labov 1972), ebenso Strategien der Aufschaukelung und Abwiegelung und schließlich die bei der Gestaltung und der Vermeidung von Streit wirksamen Kommunikationsprinzipien. Für die stärker argumentativ orientierten Streitformen wären Beobachtungen zur Eristik heranzuziehen (z. B. Schopenhauers „Eristische Dialektik“).

Besser ist die Forschungslage in bezug auf die Geschichte von Komplimentsequenzen. In Arbeiten wie Braungart (1988), Beetz (1990) und Fauser (1991) sind wichtige Grundmuster des Komplimentierens und deren Funktion im Überblick beschrieben. Es werden Entwicklungstendenzen vom 16.-18. Jahrhundert erkennbar gemacht und mit Veränderungen der Lebensform in Zusammenhang gebracht. Was aus dialoganalytischer Sicht bisher noch unterrepräsentiert ist, ist die Rekonstruktion von Typen von Dialogverläufen, alternativen Strategien und charakteristischen Äußerungsformen. Hier gibt es für die gegenwärtige Praxis des Komplimentemachens, vor allem in angelsächsischen Ländern, methodisch lehrreiche Beschreibungen (vgl. z. B. Herbert 1989).

Eine der möglichen Veränderungen von Dialogformen besteht darin, daß bestimmte Strategien besonders hervorgehoben werden, sei es daß sie besonders positiv bewertet, besonders kritisch betrachtet oder zu einem eigenen Spiel ausgebildet werden (vgl. Carlson 1983, 56f.). Was die Bewertung von einzelnen Handlungen und Strategien angeht, so werden einige Hinweise im Abschnitt über Kommunikationsprinzipien (3.5) gegeben. Hier soll kurz ein Beispiel dafür angeführt werden, wie sich eine bestimmte Strategie zu einem Spiel sui generis entwickeln kann. In alltäglichen Planungskommunikationen besteht eine gebräuchliche Strategie darin, Vorschläge zu machen und diese dann gleich zu diskutieren, um dann zu anderen, möglicherweise modifizierten Vorschlägen überzugehen und diese auf dieselbe Art und Weise zu behandeln. Diese 
Strategie hat den Vorteil, daß man sich frühzeitig über die Qualität der gemachten Vorschläge Rechenschaft ablegt, sie hat aber den Nachteil, daß erst nach längerem Dialogverlauf eine größere Zahl von Vorschlägen auf den Tisch kommt. Möchte man in der explorativen Phase einer Problemlösekommunikation eine möglichst hohe Zahl unterschiedlicher Vorschläge und sonstiger relevanter Hinweise erzielen, so kann man die Strategie favorisieren oder gar vorschreiben, daß Vorschläge und sonstige Hinweise zunächst undiskutiert aufgehäuft werden. Diese Strategie wurde in den 30er Jahren in den USA entwickelt und als brainstorming bezeichnet. Brainstorming ist also eine Einzelstrategie von Planungs- bzw. Problemlösedialogen, die zu einer eigenen Dialogform weiterentwickelt wurde. (Zu Sequenzen und Strategien in Planungsdialogen vgl. Fritz 1982, Kap. 8.)

\subsection{Institutionen und Sprecherkonstellationen}

Eine Institution kann man definieren als eine Struktur von Handlungsmustern, nach denen bestimmte Typen von Personen handeln und über die gemeinsames Wissen besteht (vgl. Berger/Luckmann 1976, 72ff.; vgl. auch Art. 16). In diesem Sinne verstanden, sind Dialogformen selbst institutionsartige Strukturen, allerdings mit der Einschränkung, daß hier nicht notwendigerweise eine Spezialisierung bestimmter Dialogteilnehmer auf bestimmte sprachliche Handlungsmuster vorausgesetzt ist.

Institutionen in einem engeren Sinne sind Organisationsformen wie Familie, Arbeitsplatz, Wochenmarkt, vor allem aber, auf der Makroebene der Beschreibung einer Gesellschaft, Formen wie Verwaltungseinrichtungen, Religionsgemeinschaften, Wissenschaftseinrichtungen und Medien. In Institutionen dieser Art entwickeln sich häufig standardisierte Sonderformen der alltäglichen Dialogformen, man denke z.B. an Formen von Lehr- und Lernkommunikationen in Schulen und an Universitäten (z. B. Vorlesung, Disputation, Seminar; vgl. Paulsen 1919), an Sonderformen von Bittgesprächen in der Audienz bei Hofe (sog. Intercessionen), an Frage/Antwort-Kommunikationen bei der Visite in der Klinik (vgl. Bliesener 1982) oder an Sonderformen des Trostgesprächs im seelsorgerlichen Gespräch usw. Wie die obige Definition von Institution schon nahelegt, bilden sich in Institutionen standardisierte Sprecherkonstellationen heraus, die durch Kompetenz- und Wissensverteilung und durch Handlungsspezialisierungen für einzelne Sprechertypen gekennzeichnet sind. Eine Möglichkeit, derartige Konstellationen zu beschreiben, ist die Annahme von institutionsspezifischen Rollen. Eine Rolle in einer Dialogform kann man definieren als die geordnete Menge der Muster, nach der ein bestimmter Dialogteilnehmer in Dialogen bestimmter Art handeln kann (vgl. Heringer 1974, 69). Inhaber/innen einer speziellen Rolle innerhalb einer Institution sind also für bestimmte (sprachliche) Handlungen zuständig. In vielen Fällen geht diese Zuständigkeit mit bestimmten Privilegien einher, u.a. beim turn-taking. Beispiele für solche Rollen sind Priester, Schamanen, Lehrer, Anwälte, professionelle Berater, Psychoanalytiker, Moderatoren, Hofnarren usw. (Zur Rolle der Schamanen vgl. Eliade 1968; zur Geschichte der Hofnarren vgl. Lever 1983.) Die Geschichte der jeweiligen Rolle ist aufs engste verknüpft mit der Geschichte der 
Institution, so daß sich wesentliche Aspekte der Institutionsgeschichte in der Geschichte der Rolle zeigen. Probleme, die einzelne Rollenträger mit der Gestaltung der Rolle haben, können Indikatoren sein für historische Veränderungen der Rolle und ihres institutionellen Zusammenhangs.

\section{3 Äußerungsformen}

Zur Geschichte der Äußerungsformen unter dialoganalytischem Aspekt gehören groBe Teile der historischen Grammatik und Semantik (bzw. der Geschichte des Wortschatzes). Einen besonderen Schwerpunkt bildet die Geschichte der gesprochenen Sprache (zur heutigen gesprochenen Sprache vgl. Art. 2). Dabei gibt es natürlich Gegenstandsbereiche, die aufgrund der Quellenlage vor allem für die neueste Zeit aussichtsreich zu bearbeiten sind, z. B. Entwicklungen der phonetischen Realisierung (vgl. aber Weithase 1961 zur Sprecherziehung im 19. Jahrhundert, Bellmann $1990 \mathrm{zu}$ Bemühungen um ,schriftgemäße Aussprache“ seit dem 16. Jahrhundert), insbesondere Entwicklungen der Intonationspraxis. Besser sieht es für eine über weitere Zeiträume ausgreifende Forschung im Bereich der Syntax aus (vgl. z. B. Sandig 1973; Grosse 1985; Betten 1990; Sonderegger 1980; 1981; 1990). Syntaktische Kurzformen, Satzarten, Wortstellungsvarianten, der Gebrauch von Anakoluthen, der Gebrauch von Modalpartikeln, Mittel der Redewiedergabe (z.B. Konjunktivgebrauch) - um nur einige Beispiele zu nennen - sind historisch nicht invariant. Für die Möglichkeiten der Ellipse zeigt das der Vergleich der folgenden mhd. Sequenz (Nibelungenlied 872f.) mit ihrem nhd. Gegenstück:

(1) A: ob er sin innen wurde, so torste in niemen bestan.

B: Nein er.

(2) A: Wenn er es merkt, kann sich keiner an ihn heranwagen.

B: Das wird er nicht.

Für viele sprachliche Handlungen gibt es syntaktisch und lexikalisch standardisierte Äußerungsformen. Dies gilt zunächst einmal für routinisierte Sprechhandlungsmuster wie Anrede-, Gruß-, Fluch-, Gratulations- und Wunschhandlungen.

(Zu Routineformeln in der Kommunikation vgl. Coulmas 1981. Zur Geschichte der Anredeformen im Dt. vgl. Metcalf 1938; Fritz/Muckenhaupt 1984; 189ff.; zu Grußformeln im Afrz. vgl. Lebsanft 1988; 1989; zur Geschichte des Fluchens im Engl. vgl. Hughes 1991, im Schweizerdeutschen vgl. Lötscher 1981.)

In diesen Bereich der verbalen Stereotype gehören auch idiomatische Wendungen, Gemeinplätze oder Sprichwörter, die für spezifische sprachliche Handlungen geeignet sind, z. B. für resümierende Abschlußhandlungen beim Erzählen („Wer zuletzt lacht, lacht am besten“). (Zur Geschichte der Sprichwörter im Dt. vgl. das Material in Wander 1867-1880.) Aber auch für weniger stereotype Handlungsmuster wie Aufforderungen, Bitten, Vorschläge etc. gibt es standardisierte Äußerungsformen. Gerade das Repertoire der Äußerungsformen für direktive Sprechakte ist in verschiedenen Sprachen und auf verschiedenen Sprachstufen funktional sehr fein ausdifferenziert 
(zur deutschen Gegenwartssprache vgl. Hindelang 1978). Hier wäre ein fruchtbares Feld für entwicklungsgeschichtliche Untersuchungen. So gibt es im Deutschen sog. modalisierte Sprechakte schon seit dem Mhd. (,ih wil iu daz raten“, ,Das möchte ich euch raten'), jedoch ändert sich der Formenbestand im Laufe der Zeit. Die Form „Darf ich Sie bitten,... ?" scheint sich im Deutschen erst gegen Ende des 17. Jahrhunderts zu finden, die Form „Ich darf Sie bitten, ..." erst in neuerer Zeit.

In bezug auf den lexikalischen Bestand gälte es einerseits, den Teil des Wortschatzes zu untersuchen, der für den illokutionären Aspekt sprachlicher Handlungen eine wichtige Rolle spielt, von den handlungskennzeichnenden Ausdrücken in performativen Formeln (Ich verspreche dir...) über handlungsspezifische Ausdrücke wie etwa Schimpfwörter (vgl. z.B. Lepp 1908; Maas 1952; Bering 1978; Hughes 1991) und bewertende Ausdrücke (z. B. klug, weise etc.; vgl. Trier 1931) bis hin zu sog. Modalwörtern (leider, vermutlich; vgl. Schildt 1990), Modalpartikeln, epistemischen Verwendungen von Modalverben (vgl. Fritz 1991) und Interjektionen (vgl. Burger 1980). Andererseits wäre der thematische Wortschatz, der im ganzen schon besser erforscht ist (fürs Dt. vgl. etwa Maurer/Rupp 1974), unter dem Gesichtspunkt der Geschichte von Dialogthemen und deren Formulierung bzw. Realisierung aufzuarbeiten (vgl. Abschn. 3.4). Zu berücksichtigen sind auch Schichten des Wortschatzes, wie fremdsprachlicher, fachsprachlicher, regionaler, archaischer und innovativer Wortschatz, die in sich wandelnder Form für Fachgespräche, Insider-Gespräche, Lehr- und Lernkommunikationen, Dialoge mit Code-switching etc. verwendet werden können. (Zur historischen Semantik aus kommunikationsanalytischer Sicht vgl. Fritz 1988.)

\subsection{Themengeschichte}

Da Themen und thematische Zusammenhänge zu den grundlegenden Organisationsprinzipien von Dialogen gehören, ist die Themengeschichte ein integraler Bestandteil einer Geschichte von Dialogformen. Relevante Aspekte sind hier:

(i) die Distribution thematischen Wissens auf bestimmte Gruppen,

(ii) die Entdeckung, Einführung und Verbreitung von neuen Themen (z.B. Verbreitung aus dem wissenschaftlichen Diskurs in den Alltagsdiskurs; vgl. z. B. Pörksen 1986; Kalverkämper 1989),

(iii) „Themenkarrieren“ (vgl. medienwissenschaftliche Untersuchungen wie Schönbach 1982; Ehlers 1983),

(iv) die Kontinuität von Themen in bestimmten Gesellschaften bzw. Gruppen (z. B. die Tradition von religiösen Themen),

(v) Veränderungen in der Einschätzung von Themen (Themen gelten als wichtig oder unwichtig, sind ,in“ oder „out"),

(vi) Veränderungen in den Normen für den Umgang mit Themen (z. B. bei Themen wie Sexualität oder Tod).

Zwei Beispiele sollen die themenhistorische Betrachtungsweise verdeutlichen. Im Frankreich des 15. Jahrhunderts tauchen mit dem neuen Interesse für die Belange des Kindes neue Themen auf, z.B. das sexuelle Verhalten des Kindes (vgl. Ariès 1978, 182). Nun kann man erwarten, daß ein derartiges neues Thema auch neue Dialogver- 
läufe eröffnet, z.B. Argumentationen über die Frage der kindlichen Unschuld oder Erziehungsgespräche (z.B. Vorwurfsdialoge), in denen sexuelle Normen eine Rolle spielen. Ein zweites Beispiel zeigt den Zusammenhang zwischen Themengeschichte und historischer Semantik. In ihrer begriffsgeschichtlichen Untersuchung zu Emanzipation sprechen Grass/Koselleck $(1975,169)$ vom geschichtsphilosophischen Stellenwert dieses Ausdrucks ,im Argumentationshaushalt der den Ausdruck verwendenden Schichten“ und geben als Beleg für die kommunikative Brisanz des Themas Emanzipation zu Beginn des 19. Jahrhunderts folgendes Zitat: „Die Emancipation der Juden ist eine so lebhafte Tagesfrage, daß man sie kaum erwähnen kann, ohne zugleich mitten in Polemik versetzt zu sein“ (Gutzkow). Bemerkenswert ist hier die Feststellung eines engen Zusammenhangs zwischen Thema (Emanzipation) und Dialogform (Polemik).

Es ist offensichtlich, daß Themen nicht isoliert existieren, sondern in thematischen Zusammenhängen stehen (vgl. Fritz 1982, 215ff.). Thematische Zusammenhänge kann man rekonstruieren, indem man die thematischen Übergänge und Verknüpfungen in Gesprächen bestimmter Individuen oder Gruppen untersucht. Für die historische Entwicklung von Themen gilt nun dasselbe wie für die Entwicklung von illokutionären Sequenzmustern: Die Veränderung eines Themas ist - wie die Veränderung eines illokutionären Musters - eingebettet in größere Zusammenhänge, so daß eine adäquate Analyse der betreffenden Veränderung gerade darin bestehen kann, daß man die Veränderungen in den thematischen Zusammenhängen untersucht. Ein einfaches Beispiel soll das verdeutlichen. Man könnte zeigen, daß in Mitteleuropa das Thema Benzinsparen für viele Leute seit den $80 \mathrm{er}$ Jahren in thematischen Zusammenhängen steht, mit denen es im Jahre 1973, d. h. zum Zeitpunkt der ersten Ölkrise, nicht so eng verknüpft war. Schlagwortartig gesprochen, hat sich das Thema vom Zusammenhang der Energiekrise auf den Zusammenhang der Umweltzerstörung ausgedehnt, was man an charakteristischen Dialogverläufen erkennen kann. Ähnliches könnte man für viele Themen in wissenschaftlichen Gesprächen, in religösen Gesprächen usw. zeigen.

\subsection{Kommunikationsprinzipien}

In manchen philosophischen Schriften finden wir ein Bild vom Status der Kommunikationsprinzipien gezeichnet, das - aus durchaus einsichtigen theoretischen Gründen stark vereinfacht erscheint, z. B. in Grice (1975), Kasher (1976) und Hintikka (1986). Die betreffenden Autoren nehmen an, daß es einen relativ kleinen Kanon von grundlegenden Gesprächsprinzipien gibt, die von allgemeinen Rationalitätsprinzipien abgeleitet sind und universelle Gültigkeit haben. Diese Annahme erscheint unproblematisch, wenn man dabei an bestimmte Deutungen des Kooperations- oder Relevanzprinzips denkt. So könnte man die Auffassung vertreten, daß es geradezu zum Begriff des Dialogs gehört, daß die Teilnehmer ihre Beiträge wechselseitig aufeinander beziehen. In diesem Sinne sind Kooperations- und Relevanzprinzip, zumindest in der historischen Zeit, die wir überblicken, zweifellos universell gültig. Für viele andere Prinzipien ist jedoch schon aus grundsätzlichen Erwägungen heraus zu erwarten, daß sie in historisch variablen Ausprägungen und mit unterschiedlichen „Ausführungsbestim- 
mungen" angewendet werden. Da sich Rationalität in der Anwendung geeigneter Mittel für spezifische Zwecke zeigt, und da sich die in der Kommunikation verfolgten Zwecke historisch verändern und dementsprechend die Dialogteilnehmer auch wechselnde kollektive Erfahrungen mit bestimmten Kommunikationssituationen machen, ist damit zu rechnen, daß auch die relevanten Prinzipien der Veränderung unterliegen bzw. zu verschiedenen Zeiten unterschiedliches Gewicht haben. Diese Überlegungen haben ein direktes Gegenstück in ethnographischen Reflexionen zur Universalität von Rationalitäts- und Kommunikationsprinzipien (vgl. Winch 1972, 34f.; Keenan 1976). Genau wie dort wird man in der Geschichte von Dialogformen die Universalität der Geltung von Prinzipien nicht ungeprüft voraussetzen, sondern man wird durch historische Analysen zu zeigen versuchen, in welchem Maß die Prinzipien historische Kontinuität oder Veränderung, Einheitlichkeit oder Vielfalt der Ausprägungen erkennen lassen.

Beispiele für z. T. konkurrierende Kommunikationsprinzipien (bzw. deren Anwendungen), die einer genetischen Erklärung bedürfen, finden sich in großer Zahl. Es sollen hier nur einige Beispiele angeführt werden:

(i) Das Prinzip der Schweigsamkeit in mönchischen Traditionen (z. B. Benediktinerregel 7, 56ff.; vgl. Hahn 1991; Luhmann/Fuchs 1989, 21ff.) und das Prinzip der Gesprächigkeit („Konversabilität") in höfischen Konversationslehren (vgl. Strosetzki 1978, 55);

(ii) das Gricesche Prinzip der Informativität und das auf Madagaskar beobachtete Prinzip der Informationszurückhaltung (Keenan 1976);

(iii) das Prinzip der Höflichkeit in seinen wechselnden Ausprägungen und Ausdrucksformen (vgl. Brown/Levinson 1987; Beetz 1990), auch in den ironischen Formulierungen grobianischer Texte;

(iv) das Prinzip der Kürze in der höfischen Rede und seine unterschiedliche Begründung für Fürsten und Diener (Kürze als Majestätsbeweis vs. Kürze als Ehrfurchtsbeweis; vgl. Braungart 1988, 18).

(v) das perspicuitas-Prinzip der klassischen Rhetorik - eine traditionelle Version des Verständlichkeitsprinzips - und die „Stilistik des Verschleierns und Enträtselns“ in der höfischen Konversationskunst des 17. Jahrhunderts (vgl. Schmölders 1986, 29; zur Geschichte des Verständlichkeitsprinzips vgl. auch Göttert 1991).

Zeugnisse für spezifischere Prinzipien, ihre Konkurrenten und Anwendungen sind:

(vi) Das Frageverbot (,ir ensult niht vil gevragen“, „Gurnemanz' Rat“; Parzival 171, 17) und sein zeitgenössisches Gegenprinzip („Frage und wisiu lere, die bringent michel ere"; Freidank 78, 23f.);

(vii) das seit der Antike immer wieder ausgesprochene Selbstlobverbot und die akzeptierte Praxis des Selbstlobs bei den Trobriandern (vgl. Malinowski 1923) und den germanischen Kriegern;

(viii) die Ächtung der Schmeichelei in Theophrasts „Charakteres“ (und der umfangreichen Theophrast-Rezeption) und die Hochschätzung maßvollen Lobs bei manchen Autoren (z. B. Hunold 1716, 82f.)

(ix) die Hochachtung des Kompliments im höfischen Diskurs des 17. Jahrhunderts und die z.T. schon zeitgenössische, aber im 18. Jahrhundert verstärkte Kritik am Komplimentierwesen (vgl. Beetz 1990; Fauser 1991, 241ff.). 
Dies ist nur eine kleine Auswahl von Prinzipien, die deutlich macht, in welchem Maß die Gültigkeit und Relevanz bestimmter Prinzipien von historischen Besonderheiten der Lebensform, des Menschenbildes und der Institutionen abhängt. Die Thematisierung von Kommunikationsprinzipien kann ein Indikator für kommunikative Probleme sein, die in einer bestimmten Zeit aktuell sind, bzw. dafür, daß es eine Praxis gibt, diese Prinzipien gerade nicht zu befolgen. Allerdings muß man immer damit rechnen, daß in bestimmten Traditionen Kommunikationsprinzipien weitergereicht werden, ohne daß damit ein direkter Bezug zu aktuellen Problemen verbunden sein muß. Dies gilt z.B. ebenso für die Übernahme antiker Prinzipien in Schriften der Humanisten (z. B. das Schmeicheleiverbot) wie für die Tradition der Kritik an sophistischen Winkelzügen beim Argumentieren bis ins 19. Jahrhundert (vgl. Hamblin 1970).

Um sich ein Bild von dem Aspektreichtum historischer Kommunikationsprinzipien zu verschaffen, kann man zunächst einmal die Anwendungsbereiche der Prinzipien unterscheiden. Prinzipien beziehen sich auf einzelne Handlungsmuster (z. B. das Fragen, das Fluchen, das Lügen, das Widersprechen), auf den Sprecherwechsel (z. B. das Gebot, den Sprecher ausreden zu lassen), auf ganze Dialogformen (z. B. das Argumentieren, das Komplimentieren, den Klatsch), auf Themen, die in bestimmten Kontexten als geeignet oder anrüchig gelten (das Wetter, bestimmte Körperfunktionen), und auf Äußerungsformen, die in bestimmten Kontexten angeraten oder verpönt sind (Höflichkeitsformen, Vulgarismen, Regionalismen, komplexe syntaktische Muster). Prinzipien sind bisweilen spezifiziert für bestimmte Personengruppen (Kinder, Frauen, Geistliche) oder Praxiszusammenhänge (z. B. das Essen, bei dem man nach Auskunft mittelalterlicher Tischzuchten nicht reden soll oder wenigstens nichts Schlechtes über die Speisen oder den Gastgeber sagen soll; vgl. Elias 1978). Und sie können spezifiziert sein nach bestimmten Wertbereichen. Neben kommunikationsmoralischen Prinzipien („Du sollst nicht lügen“) und ästhetischen Prinzipien („Man soll lautreines Deutsch sprechen") finden wir strategische Prinzipien, in denen sich Erfolgs- und Mißerfolgserfahrungen kristallisieren (,Bei Verhandlungen sollte man möglichst wenig Information herausrücken“; vgl. Francis Bacons Essay „Of negotiating“ von 1597). Schließlich finden wir, als Inkarnation bestimmter Typen von Prinzipienverletzung, in der Literatur ebenso wie im alltäglichen Gespräch, die Beschreibung von kommunikativen Charakteren, wie den Schwätzer, den Schmeichler, den Heuchler oder das Klatschmaul (zum Klatsch vgl. Bergmann 1987).

Als Quellen für die Geschichte von Kommunikationsprinzipien bieten sich zunächst einmal Texte an, in denen derartige Prinzipien thematisiert werden, d.h. vor allem dialogkritische und dialogpädagogische Schriften bzw. entsprechende Passagen aus Texten unterschiedlicher Art, sei es literarischer, religiöser, (auto)biographischer oder pädagogischer Natur. Bei der Analyse dieser Quellen ist es von besonderem Interesse herauszufinden, wer die Prinzipien für wen formuliert, wie die Prinzipien legitimiert werden, ob konkurrierende Prinzipien erwähnt werden, ob es Systeme von Prinzipien (ein Kommunikationsideal, eine Kommunikationsmoral) oder Hierarchien von Prinzipien gibt, welche Indizien es für Verletzungen der betreffenden Prinzipien gibt, für welche Dialogformen oder Situationen die Prinzipien spezifiziert werden und in wel- 
cher Weise die Form der Anwendung von Prinzipien konkretisiert wird. Neben den erwähnten Quellenarten kommen natürlich alle möglichen Dialogdokumente, authentische und fiktionale, als Quellen infrage, wobei es allerdings oft schwierig ist, aus einem vorliegenden Dialogdokument darauf zu schließen, welche Prinzipien hier implizit im Spiel sind bzw., bei fiktionalen Darstellungen, welche Befolgungen und Nichtbefolgungen ein Autor darstellen will und warum. Hier liegen besonders heikle hermeneutische Probleme, die u.a. in der Gefahr bestehen, daß man ungeprüft die Gültigkeit von uns vertrauten Prinzipien unterstellt.

\section{Literaturhinweise}

Ariès, P. (1978): Geschichte der Kindheit. München.

Bauman, R./Sherzer, J. (eds.) (1974): Explorations in the ethnography of speaking. Cambridge.

Bax, M. (1991): Historische Pragmatik: Eine Herausforderung für die Zukunft. Diachrone Untersuchungen zu pragmatischen Aspekten ritueller Herausforderungen in Texten mittelalterlicher Literatur. In: Busse, D. (Hg.): Diachrone Semantik und Pragmatik. Untersuchungen zur Erklärung und Beschreibung des Sprachwandels. Tübingen, 197-215.

Bebermeyer, G. (1984): Streitgedicht/Streitgespräch. In: Reallexikon der deutschen Literaturgeschichte. 2. Aufl. Bd. 4. Berlin/New York, 225-245.

Beetz, M. (1990): Frühmoderne Höflichkeit. Komplimentierkunst und Gesellschaftsrituale im altdeutschen Sprachraum. Stuttgart.

Bellmann, G. (1990): Eine Quelle der deutschen Sprachgeschichte des 17. und 18. Jahrhunderts. In: Besch, W. (Hg.): Deutsche Sprachgeschichte. Grundlagen, Methoden, Perspektiven. Frankfurt a. M., 289-300.

Berger, D. A. (1978): Die Konversationskunst in England 1660-1740. München.

Berger, P.L./Luckmann, Th. (1967): The social construction of reality. Harmondsworth.

Bergmann, J. R. (1987): Klatsch. Zur Sozialform der diskreten Indiskretion. Berlin/New York.

Bering, D. (1978): Die Intellektuellen. Geschichte eines Schimpfwortes. Stuttgart.

Betten, A. (1985): Sprachrealismus im deutschen Drama der siebziger Jahre. Heidelberg.

Betten, A. (1990): Zur Problematik der Abgrenzung von Schriftlichkeit und Mündlichkeit bei mittelalterlichen Texten. In: Betten, A. (Hg.): Neuere Forschungen zur historischen Syntax des Deutschen. Tübingen, 234-335.

Bliesener, T. (1982): Die Visite - ein verhinderter Dialog. Initiativen von Patienten und Abweisungen durch das Personal. Tübingen.

Bömer, A. (1897/1899): Die lateinischen Schülergespräche der Humanisten. Auszüge mit Einleitungen, Anmerkungen und Namen- und Sachregister. 2 Teile. Berlin.

Braungart, G. (1988): Hofberedsamkeit. Studien zur Praxis höfisch-politischer Rede im deutschen Territorialabsolutismus. Tübingen.

Brown, P./Levinson, S. C. (1987): Politeness: Some universals in language usage. Cambridge.

Burger, H. (1980): Interjektionen. In: Sitta, H. (Hg.): Ansätze zu einer pragmatischen Sprachgeschichte. Tübingen, 53-70.

Carlson, L. (1983): Dialogue games. An approach to discourse analysis. Dordrecht/Boston/ London.

Coulmas, F. (1981): Routine im Gespräch. Zur pragmatischen Fundierung der Idiomatik. Wiesbaden.

Ehlers, R. (1983): Themenstrukturierung durch Massenmedien. Zum Stand der empirischen Agenda-Setting-Forschung. In: Publizistik 27, 167-186. 
Eliade, M. (1968): Le schamanisme. 2e éd. Paris.

Elias, N. (1978): Über den Prozeß der Zivilisation. 2 Bde. 5. Aufl. Frankfurt a. M. (1. Aufl. 1939).

Fauser, M. (1991): Das Gespräch im 18. Jahrhundert. Rhetorik und Geselligkeit in Deutschland. Stuttgart.

Fritz, G. (1982): Kohärenz. Grundfragen der linguistischen Kommunikationsanalyse. Tübingen.

Fritz, G. (1988): Change of meaning and change of vocabulary. In: Sociolinguistics. An international handbook of the science of language and society. Vol. II. Berlin/New York, 1614-1631.

Fritz, G. (1991): Deutsche Modalverben 1609: Epistemische Verwendungsweisen. In: Beiträge zur Geschichte der deutschen Sprache und Literatur 113, 28-52.

Fritz, G./Muckenhaupt, M. (1984): Kommunikation und Grammatik. 2. Aufl. Tübingen.

Geertz, C. (1983): Dichte Beschreibung. Beiträge zum Verstehen kultureller Systeme. Frankfurt a. M.

Gloning, Th. (1993): Sprachreflexive Textstellen als Quellen für die Geschichte von Kommunikationsformen. In: Löffler, H. (Hg.): Dialoganalyse IV. Tübingen.

Goffman, E. (1971): Relations in public. Microstudies of the public order. Harmondsworth.

Göttert, K.-H. (1988): Kommunikationsideale. Untersuchungen zur europäischen Konversationstheorie. München.

Göttert, K.-H. (1991): Ringen um Verständlichkeit. Ein historischer Streifzug. In: Deutsche Vierteljahrsschrift für Literaturwissenschaft und Geistesgeschichte 65, 1-14.

Grass, K. M./Koselleck, R. (1975): Emanzipation. In: Brunner, O./Conze, W./Koselleck, R. (Hg.): Geschichtliche Grundbegriffe, Historisches Lexikon zur politisch-sozialen Sprache in Deutschland. Bd. 2. E-G. Stuttgart, 153-197.

Grice, H. P. (1975): Logic and conversation. In: Cole, P./Morgan, J. L. (eds.): Syntax and semantics. Vol. 3: Speech acts. New York, 41-58.

Grosse, S. (1985): Reflexe gesprochener Sprache im Mittelhochdeutschen. In: Sprachgeschichte. Ein Handbuch zur Geschichte der deutschen Sprache und ihrer Erforschung. 2. Halbbd. Berlin/ New York, 1186-1191.

Grünert, H. (1974): Sprache und Politik. Untersuchungen zum Sprachgebrauch der ,Paulskirche'. Berlin.

Gumperz, J. J./Hymes, D. (eds.) (1972): Directions in sociolinguistics. The ethnography of communication. New York.

Hahn, A. (1991): Rede- und Schweigeverbote. In: Kölner Zeitschrift für Soziologie 43, 86-105.

Henne, H. (1980): Probleme einer historischen Gesprächsanalyse. Zur Rekonstruktion gesprochener Sprache im 18. Jahrhundert. In: Sitta,H. (Hg.): Ansätze zu einer pragmatischen Sprachgeschichte. Tübingen, 89-102.

Herbert, R. (1989): The ethnography of English compliment and compliment responses: A contrastive sketch. In: Oleksy, W. (ed.): Contrastive pragmatics. Amsterdam/Philadelphia, 3-36.

Heringer, H. J. (1974): Praktische Semantik. Stuttgart.

Heringer, H. J. (1977): Sprachspiele und ihre Geschichte. In: Heringer, H. J. u. a.: Einführung in die praktische Semantik. Heidelberg, 60-85.

Heusler, A. (1969): Der Dialog in der altgermanischen erzählenden Dichtung. In: Kleine Schriften. Bd. 2. Berlin 1969, 611-689.

Hindelang, G. (1978): Auffordern. Die Untertypen des Aufforderns und ihre sprachlichen Realisierungsformen. Göppingen.

Hirzel, R. (1895): Der Dialog. Ein literarhistorischer Versuch. 2 Bde. Leipzig.

Holly, W. (1982): Zur Geschichte parlamentarischen Sprachhandelns in Deutschland. Eine historisch-pragmatische Skizze an Beispielen aus ersten Sitzungen von verfassungsgebenden Versammlungen. In: Zeitschrift für Literaturwissenschaft und Linguistik 47, 10-48.

Hughes, G. (1991): Swearing: a social history of foul language, oaths and profanity in English. Oxford.

Hunold, Christian Friedrich (1716): Die Beste Manier In Honneter Conversation, Sich höflich und Behutsam aufzuführen / Und in kluger Conduite zu leben. Von Menantes. Hamburg. 
Kästner, H. (1978): Mittelalterliche Lehrgespräche. Textlinguistische Analyse. Studien zur poetischen Funktion und pädagogischen Intention. Berlin.

Kalverkämper, H. (1989): Kolloquiale Vermittlung von Fachwissen im frühen 18. Jahrhundert. Gezeigt an den „Entretiens sur la Pluralité des Mondes“ (1686) von Fontenelle. In: SchliebenLange, B. (Hg.): Fachgespräche in Aufklärung und Revolution. Tübingen, 17-80.

Kasher, A. (1976): Conversational maxims and rationality. In: Kasher, A. (ed.): Language in focus: Foundations, methods and systems. Dordrecht, 197-216.

Kasher, A. (1986): Politeness and rationality. In: Pragmatics and linguistics. Festschrift Jacob L. Mey. Odense, 103-114.

Katz, D./Katz, R. (1928): Gespräche mit Kindern. Berlin.

Keenan, E. O. (1976): The universality of conversational postulates. In: Language in Society 5, 67-80.

Keller, R. (1990): Sprachwandel. Von der unsichtbaren Hand in der Sprache. Tübingen.

Knigge, A. Freiherr v. (1790): Über den Umgang mit Menschen. 3. Aufl. Hannover. Hg. v. G. Ueding. Frankfurt a.M. 1977.

Köhler, E. (1968): „Conseil des barons“ und ,jugement des barons“. Epische Fatalität und Feudalrecht im altfranzösischen Rolandslied. In: Sitzungsberichte der Heidelberger Akademie der Wissenschaften. Phil.-hist. Klasse. 52. Jahrgang. 4. Abhandlung.

Kornblum, U. (1971): Beweis. In: Handwörterbuch zur deutschen Rechtsgeschichte. Bd. 1. Berlin, Sp. 401-408.

Labov, W. (1972): Rules for ritual insults. In: Sudnow, D. (ed.): Studies in social interaction. New York, $120-169$.

Lebsanft, F. (1988): Studien zu einer Linguistik des Grusses. Sprache und Funktion der altfranzösischen Grußformeln. Tübingen.

Lebsanft, F. (1989): Kontinuität und Diskontinuität antiker Rede- und Grußformen. Aspekte der Sprach- und Gesellschaftskritik. In: Erzgräber, W. (Hg.): Kontinuität und Transformation der Antike im Mittelalter. Sigmaringen, 285-299.

Lepp, F.: Schlagwörter des Reformationszeitalters. Leipzig 1908.

Lever, M. (1983): Le sceptre et la marotte. Paris.

Lewis, D. K. (1969): Convention. A philosophical study. Cambridge, Mass.

Lötscher, A. (1981): Zur Sprachgeschichte des Fluchens und Beschimpfens im Schweizerdeutschen. In: Zeitschrift für Dialektologie und Linguistik 48, 145-160.

Luckmann, Th. (1986): Grundformen der gesellschaftlichen Vermittlung des Wissens: Kommunikative Gattungen. In: Neidhardt, F. u. a. (Hg.): Kultur und Gesellschaft. Sonderheft 27 der Kölner Zeitschrift für Soziologie und Sozialpsychologie. Opladen, 191-211.

Luhmann, N./Fuchs, P. (1989): Reden und Schweigen. Frankfurt a. M.

Maas, H. (1952): Das Nürnberger Scheltwort. In: Mitteilungen des Vereins für Geschichte der Stadt Nürnberg 43, 361-483.

Maurer, F./Rupp, H. (Hg.) (1974/1978): Deutsche Wortgeschichte. 3 Bde. Berlin/New York.

Naumann, B. (1991): „Merkt euch dieses, meine Lieben!“ Der didaktische Dialog in Joachim Heinrich Campes „Robinson der Jüngere“ (1779). In: Stati, S. u. a. (Hg.): Dialoganalyse III. Bd. 1. Tübingen, 377-389.

Ong, W. J. (1958): Ramus, method, and the decay of dialogue: From the art of discourse to the art of reason. Cambridge, Mass.

Paulsen, F. (1919): Geschichte des gelehrten Unterrichts auf den deutschen Schulen und Universitäten vom Ausgang des Mittelalters bis zur Gegenwart. 2 Bde. 3. Aufl. Leipzig.

Pörksen, U. (1986): Deutsche Naturwissenschaftssprachen. Historische und kritische Studien. Tübingen.

Sandig, B. (1973): Zur historischen Kontinuität normativ diskriminierter syntaktischer Muster in spontaner Sprechsprache. In: Deutsche Sprache 1, 37-57.

Schlieben-Lange, B. (1979): Ai las - que planhs? Ein Versuch zur historischen Gesprächsanalyse am Flamenca-Roman. In: Romanistische Zeitschrift für Literaturgeschichte, 1-30. 
Schlieben-Lange, B. (1983): Traditionen des Sprechens. Elemente einer pragmatischen Sprachgeschichtsschreibung. Stuttgart.

Schmölders, C. (Hg.) (1986): Die Kunst des Gesprächs. Texte zur Geschichte der europäischen Konversationstheorie. Mit einer Einleitung. 2. Aufl. München.

Schönbach, K. (1982): ,The issues of the seventies'. Elektronische Inhaltsanalyse und die langfristige Beobachtung von Agenda-Setting-Wirkungen der Massenmedien. In: Publizistik 28, $129-140$.

Schopenhauer, A. (1830): Eristische Dialektik. In: Der handschriftliche Nachlaß. Hg, von A. Hübscher. Bd. 3, Berliner Manuskripte (1818-1830). München 1985, 666-695.

Schwarz, A. (1984): Sprechaktgeschichte. Studien zu den Liebeserklärungen in mittelalterlichen und modernen Tristan-Dichtungen. Göppingen.

Schwitalla, J. (1986): Martin Luthers argumentative Polemik: mündlich und schriftlich. In: Worstbrock, F.J./Koopmann, H. (Hg.): Kontroversen, alte und neue. Band 2. Tübingen, $41-54$.

Sonderegger, S. (1980): Gesprochene Sprache im Althochdeutschen und ihre Vergleichbarkeit mit dem Neuhochdeutschen. Das Beispiel Notkers des Deutschen von St. Gallen. In: Sitta, H. (Hg.): Ansätze zu einer pragmatischen Sprachgeschichte. Tübingen, 71-88.

Sonderegger, S. (1981): Gesprochene Sprache im Nibelungenlied. In: Masser, A. (Hg.): Hohenemser Studien zum Nibelungenlied. Dornbirn, 360-381.

Sonderegger, S. (1990): Syntaktische Strukturen gesprochener Sprache im älteren Deutschen. In: Betten, A. (Hg.): Neuere Forschungen zur historischen Syntax des Deutschen. Tübingen, 310-323.

Schildt, J. (1990): Modalwörter im Frühneuhochdeutschen. Die Entwicklung ihres Bestandes. In: Betten, A. (Hg.): Neuere Forschungen zur historischen Syntax des Deutschen. Tübingen, $152-162$.

Strecker, B. (1987): Strategien des kommunikativen Handelns. Düsseldorf.

Strosetzki, Ch. (1978): Konversation. Ein Kapitel gesellschaftlicher und literarischer Pragmatik im Frankreich des 17. Jahrhunderts. Frankfurt a. M./Bern/Las Vegas.

Toulmin, S. (1972): Human understanding. The collective use and evolution of concepts. Princeton, New Jersey.

Trier, J. (1931): Der deutsche Wortschatz im Sinnbezirk des Verstandes. Bd. 1. Von den Anfängen bis zum Beginn des 13. Jahrhunderts. Heidelberg.

Ullman-Margalit, E. (1978): Invisible-hand explanations. Synthese 39, 263-291.

Wander, K. F. W. (1867-1880): Deutsches Sprichwörter-Lexikon. Leipzig.

Weigand, E. (1988): Historische Sprachpragmatik am Beispiel: Gesprächsstrukturen im Nibelungenlied. In: Zeitschrift für deutsches Altertum und deutsche Literatur 117, 159-173.

Weithase, I. (1961): Zur Geschichte der gesprochenen deutschen Sprache. 2 Bde. Tübingen 1961.

Winch, P. (1972): Understanding a primitive society. In: Winch, P.: Ethics and action. London, $8-49$.

Gerd Fritz, Gießen 


\section{Handbuch der Dialoganalyse}

herausgegeben von

Gerd Fritz und Franz Hundsnurscher

Max Niemeyer Verlag

Tübingen 1994 